Supporting Information

\title{
Molecular Weight Effect on the Transition Processes of a Symmetric PS- $b$-P2VP during Spin-Coating
}

Hiroki Ogawa ${ }^{1,2}$, Mikihito Takenaka ${ }^{1,2}$, Tsukasa Miyazaki ${ }^{3}$

${ }^{1}$ Institute for Chemical Research, Kyoto University, Gokasho, Uji, Kyoto 611-0011, Japan

${ }^{2}$ Riken SPring-8 Center, 1-1-1 Kouto, Sayo, Hyogo 679-5148, Japan

${ }^{3}$ Comprehensive Research Organization for Science and Society, Shirakata, Tokai, Ibaraki 319-1106, Japan

\section{Self-assembled nanostructures after spin-coating}

Figure S1(a)-(e) shows the molecular weight dependence from the two-dimensional (2D) GISAXS measurements and surface height AFM images of the as-spun thin films. The GISAXS images were acquired with an exposure time of $1.0 \mathrm{~s}$. AFM was performed with a multimode JSPM-5200 scanning probe microscope AFM system (JEOL) in tapping mode. The spin-coated thin films were partially etched by an oxygen plasma to expose the P2VP domains within the thin film. In the $2 \mathrm{D}$ image of the PS- $b$-P2VP-1 (the number-average molecular weight, $M_{n}$, was 16,500), an isotropic ring pattern was observed, as shown in Figure S1(a). Figure S1(f) shows a one-dimensional (1D) profile sliced in the in-plane direction at $\mathrm{q}_{\mathrm{z}}=0.288 \mathrm{~nm}^{-1}$. We observed a broad peak at $\mathrm{q}_{\mathrm{y}} \approx 0.400 \mathrm{~nm}^{-1}$ (see the upward arrow with a line), indicating that the distinct 
ordered structure was not formed in the thin film. In the corresponding AFM image, no ordered structures were observed on the surface. Thus, the as-spun thin film of PS- $b$-P2VP-1 was in the disordered state.

Figure S1(b) shows a 2D GISAXS pattern from PS- $b$-P2VP-2 $\left(M_{n}=50,000\right)$. In addition to the isotropic ring pattern observed in the pattern, a weak streak along the $\mathrm{q}_{\mathrm{z}}$ direction was observed at $\mathrm{q}_{\mathrm{y}}=0.181 \mathrm{~nm}^{-1}$. The streak indicates that ordered structures were oriented perpendicular to the substrate in the thin film. In the corresponding AFM image, striped surface structures were observed. From these results, lamellar structures oriented perpendicular to the substrate were formed in the thin film. The $d$-spacing of the lamellar structure was estimated to be $34.7 \mathrm{~nm}$ from the peak position at $\mathrm{q}_{\mathrm{y}}=0.181$ $\mathrm{nm}^{-1}$ in the in-plane profile of Figure S1(f) (see the upward arrow with a line). A scattering peak appeared at $\left(\mathrm{q}_{\mathrm{y}}, \mathrm{q}_{\mathrm{z}}\right)=\left(0.127 \mathrm{~nm}^{-1}, 0.264 \mathrm{~nm}^{-1}\right)$ in the 2D GISAXS pattern of PS- $b$-P2VP-3 $\left(M_{n}=88,000\right)$, (Figure S1(c)). Streaks were observed in the $\mathrm{q}_{\mathrm{z}}$ direction, suggesting that the self-assembled structures were oriented perpendicular to the substrate. Dot-like structures are shown in the AFM image. For the DWBA calculation in the in-plane direction, we calculated modeling by using FitGISAXS program $^{\mathrm{S} 1}$ and also showed in Figure S1(f). We made a simple model of sandwiched islands with cylinder form factor and PY2D structural factor. $\delta$ and $\beta$ of PS and $\mathrm{P} 2 \mathrm{VP}$ are $3.440 \times 10^{-6}, 4.615 \times 10^{-9}, 3.763 \times 10^{-6}$ and $5.570 \times 10^{-9}$ respectively. We set the diameter of rod is $30.0 \mathrm{~nm}$ with the $d$-spacing of $45.0 \mathrm{~nm}$. From the calculation of the in-plane profile and AFM observation, we concluded that the cylinder structures are oriented perpendicular to the sample surface. The structures obtained at 
this molecular weight are consistent with those we previously reported, and the results are discussed in detail in that report ${ }^{35}$. The 2D GISAXS patterns of the higher molecular weight PS- $b$-P2VP-4 $\left(M_{n}=199,000\right)$ and

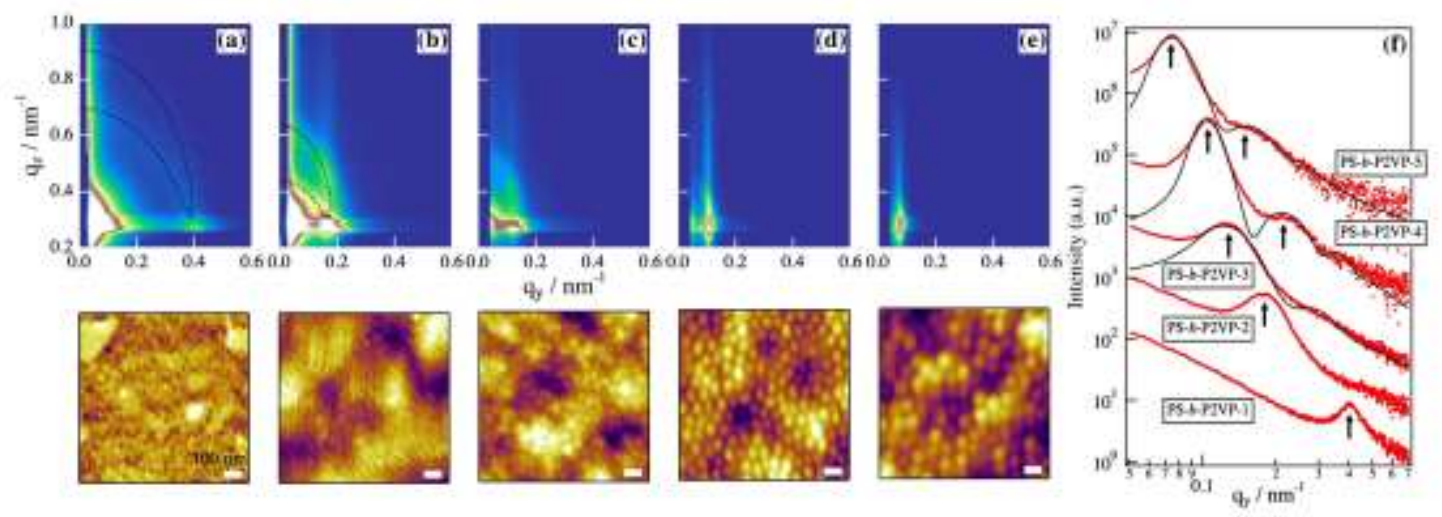

Figure S1. 2D GISAXS patterns and the corresponding surface height AFM images of spin-coated thin films for (a) PS- $b$-P2VP-1, (b) PS- $b-\mathrm{P} 2 \mathrm{VP}-2$, (c) PS- $b-\mathrm{P} 2 \mathrm{VP}-3$, (d) PS- $b$-P2VP-4, and (e) PS- $b$-P2VP-5. The incident angle of GISAXS is $0.21^{\circ}$ above the critical angle of polymers of $0.15^{\circ}$. The white parts in AFM images represent the P2VP component. The dashed and dotted lines indicate the calculated peak positions from the transmitted and reflected beams, reflectively ${ }^{\mathrm{S} 2}$. (f) The in-plane profiles of GISAXS patterns (red dots) and the calculated profile (black solid line).

the highest molecular weight PS- $b$-P2VP-5 $\left(M_{n}=428,000\right)$ showed scattering peaks at $\left(\mathrm{q}_{\mathrm{y}}, \mathrm{q}_{\mathrm{z}}\right)=\left(0.105 \mathrm{~nm}^{-1}, 0.263 \mathrm{~nm}^{-1}\right)$ and $\left(\mathrm{q}_{\mathrm{y}}, \mathrm{q}_{\mathrm{z}}\right)=\left(0.075 \mathrm{~nm}^{-1}, 0.263 \mathrm{~nm}^{-1}\right)$, respectively The peaks were observed only in the in-plane direction and extended in the $\mathrm{q}_{\mathrm{z}}$ direction, suggesting that the self-assembled structures were oriented perpendicular to the substrate (Figure S1 (d), (e)). We observed a dot-like structure on each surface by AFM. 
In each in-plane profile, we were able to observe broad secondary peaks as well as sharp primary peaks, although the secondary peaks were not observed in PS- $b$-P2VP-3. This difference indicates a higher ordering of the cylindrical structure than that of PS- $b$-P2VP-3 (see the upward arrows with lines in Figure S1 (f)). The primary peak from PS- $b-\mathrm{P} 2 \mathrm{VP}-4$ was observed around $\mathrm{q}_{\mathrm{y}}=0.105 \mathrm{~nm}^{-1}$ and that from PS- $b-\mathrm{P} 2 \mathrm{VP}-5$ was observed around $\mathrm{q}_{\mathrm{y}}=0.075 \mathrm{~nm}^{-1}$. We also made a simple model of sandwiched islands with cylinder form factor and PY2D structural factor. The calculated profile is good agreement with the in-plane profile in Figure S1(f). We set the diameter of rod is $44.0 \mathrm{~nm}$ with the $d$-spacing of $66.9 \mathrm{~nm}$ for PS- $b-\mathrm{P} 2 \mathrm{VP}-4$ and the diameter of rod is 58.0 $\mathrm{nm}$ with the $d$-spacing of $88.2 \mathrm{~nm}$ for PS- $b$-P2VP-5. These results indicated that in both samples, the cylindrical structures were oriented perpendicular to the substrate.

\section{SAXS results of the nanostructures in toluene solvent}

Small-angle X-ray scattering (SAXS) measurements were performed at the second hutch at the BL03XU beamline at SPring- 8 . The X-ray wavelength, $\lambda$, and sample-to-detector distance were set to $0.10 \mathrm{~nm}$ and $3200 \mathrm{~mm}$, respectively. The scattering images were detected by a PILATUS $1 \mathrm{M}$ (Dectris, Ltd.) apparatus with an exposure time of $1.0 \mathrm{~s}$. We measured the solution at room temperature, which is below the glass transition temperature of PS and P2VP. Figure S2 shows the SAXS profiles of the PS- $b$-P2VP with different molecular weights in toluene. To characterize the structures of the micelles, we fitted the scattering profiles by the model for the form factor of the core-shell (F(q)), and we used the Percus-Yevick (PY) hard-sphere 
interaction potential as the structure factor $(\mathrm{S}(\mathrm{q}))^{\mathrm{S3,4}}$. The solid lines correspond to the fitting results, and the model function was able to express the obtained profiles. In PS- $b$-P2VP-1, we used a model of the form factors by three different sizes (Figure $\mathrm{S} 2(\mathrm{a})$ ). We set the core radius and shell thickness of $\mathrm{F}_{1}(\mathrm{q})$ with 17.5 and $17.2 \mathrm{~nm}$, respectively; those of $F_{2}(q)$ were 7.0 and $6.5 \mathrm{~nm}$; and those of $F_{3}(q)$ were 0.9 and 0.9 $\mathrm{nm}$, respectively. In PS- $b$-P2VP-2, the form factors with three different sizes were used in Figure S2(b). The core radius and shell thickness of $F_{1}(q)$ were 7.2 and $7.1 \mathrm{~nm}$, respectively; Those values of $F_{2}(q)$ was 2.6 and $2.3 \mathrm{~nm}$; and $F_{3}(q)$ were 0.9 and $0.9 \mathrm{~nm}$, respectively. In the case of PS- $b$-P2VP-3, a model used in the best fit was $F_{1}(q) \times S_{1}(q)$ $+F_{2}(q)$, as shown in Figure $S 2(c)$. The core radius and shell thickness of $F_{1}(q)$ were 5.2 and $5.0 \mathrm{~nm}$, respectively; those of $\mathrm{F}_{2}(\mathrm{q})$ was 1.0 and $1.0 \mathrm{~nm}$, respectively. The radius of $\mathrm{S}_{1}(\mathrm{q})$ was $26.5 \mathrm{~nm}$. In PS- $b-\mathrm{P} 2 \mathrm{VP}-4$, we used a model of $\left(\mathrm{F}_{1}(\mathrm{q}) \times \mathrm{S}_{1}(\mathrm{q})\right)+\mathrm{F}_{2}(\mathrm{q})+\mathrm{F}_{3}(\mathrm{q})$ in Figure S2(d). The core radius and shell thickness of $F_{1}(q)$ were 8.2 and $7.8 \mathrm{~nm}$, respectively. The radius of $\mathrm{S}_{1}(\mathrm{q})$ was $44.0 \mathrm{~nm} . \mathrm{F}_{2}(\mathrm{q})$ was 8.5 and $10.0 \mathrm{~nm}$; and $\mathrm{F}_{3}(\mathrm{q})$ was 0.7 and $0.7 \mathrm{~nm}$, respectively. In Figure $\mathrm{S} 2(\mathrm{e})$, we used a model of $\left(\mathrm{F}_{1}(\mathrm{q}) \times \mathrm{S}_{1}(\mathrm{q})\right)+\mathrm{F}_{2}(\mathrm{q})$ for PS- $b-\mathrm{P} 2 \mathrm{VP}-5$. The core radius and shell thickness of $\mathrm{F}_{1}(\mathrm{q})$ were 18.2 and $18.5 \mathrm{~nm}$, respectively. The radius of $\mathrm{S}_{1}(\mathrm{q})$ was $150.0 \mathrm{~nm} . \mathrm{F}_{2}(\mathrm{q})$ was 1.0 and $1.0 \mathrm{~nm}$, respectively.

The results yielded the characteristic parameters for the micelles listed in Table S1. The core radius and shell thickness increased with increasing molecular weight. In the PS- $b$-P2VP-3, -4 and -5 samples, scattering peaks were observed at the positions corresponding to the intermicellar distances, whereas in the PS- $b$-P2VP-1 and -2 samples, a clear peak for intermicellar correlation was not observed. 

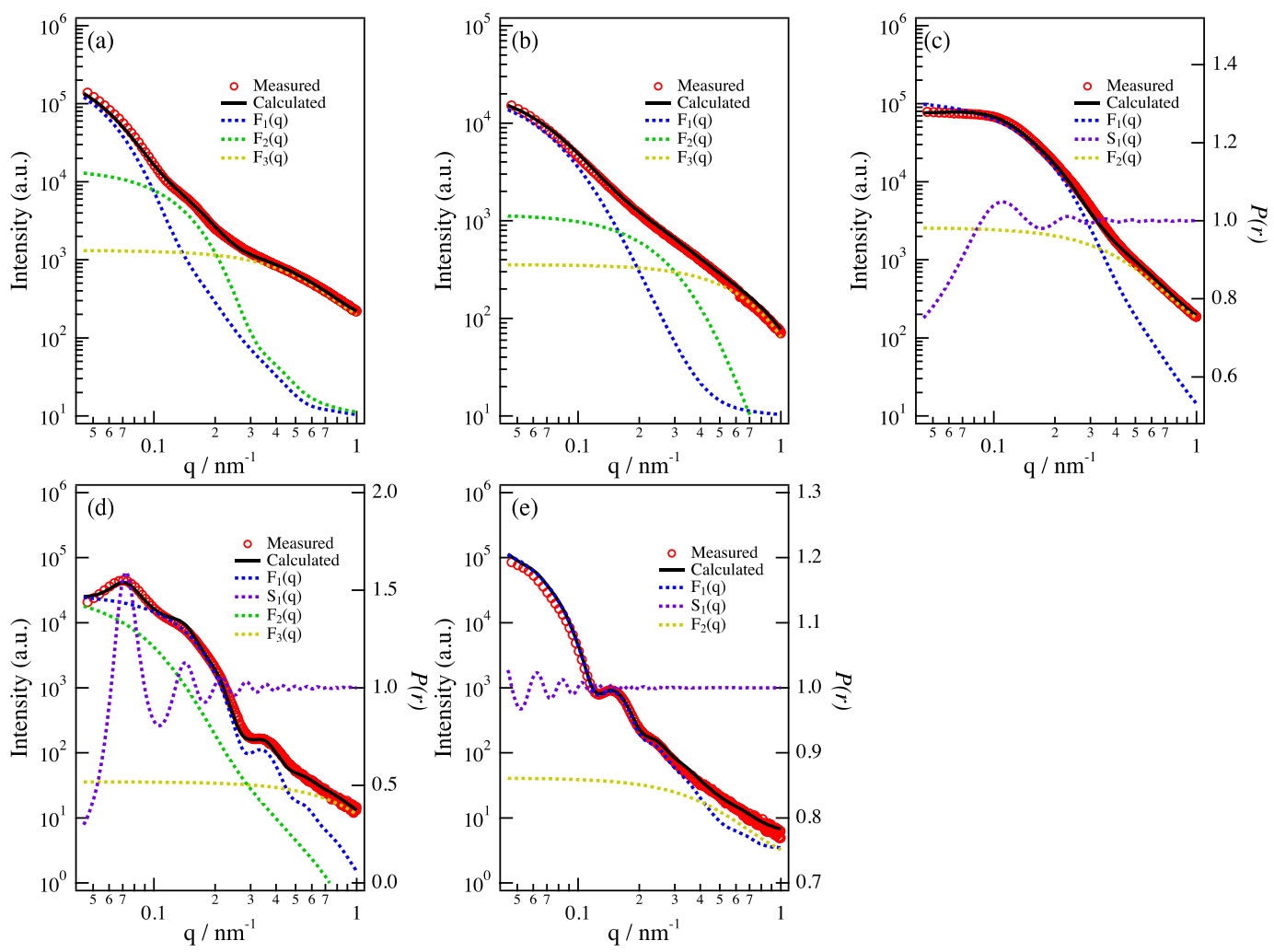

Figure S2. Measured (red circles) and calculated profiles (black solid line) with the form (blue, green yellow dashed lines) and structure factors (purple dashed lines) of (a) PS- $b$-P2VP-1, (b) PS- $b$-P2VP-2, (c) PS- $b$-P2VP-3,

(d) PS- $b-\mathrm{P} 2 \mathrm{VP}-4$ and

(e) PS- $b$-P2VP-5, respectively.

\begin{tabular}{|c|c|c|c|c|c|}
\hline & PS- $b$-P2VP-1 & PS- $b$-P2VP-2 & PS- $b$-P2VP-3 & PS- $b$-P2VP-4 & PS- $b$-P2VP-5 \\
\hline Core radius & $7.0 \mathrm{~nm}$ & $2.6 \mathrm{~nm}$ & $5.2 \mathrm{~nm}$ & $8.2 \mathrm{~nm}$ & $18.2 \mathrm{~nm}$ \\
\hline Shell thickness & $6.5 \mathrm{~nm}$ & $2.3 \mathrm{~nm}$ & $5.0 \mathrm{~nm}$ & $7.8 \mathrm{~nm}$ & $18.5 \mathrm{~nm}$ \\
\hline intermiceller distance & & & $26.5 \mathrm{~nm}$ & $44.0 \mathrm{~nm}$ & $150.0 \mathrm{~nm}$ \\
\hline
\end{tabular}

Table S1. Values from the fitting results of PS- $b$-P2VP micelles in toluene solvent at each molecular weight. 


\section{In situ GISAXS images during spin-coating}

Figure S3-S7 shows more detailed GISAXS images for each molecular weight.

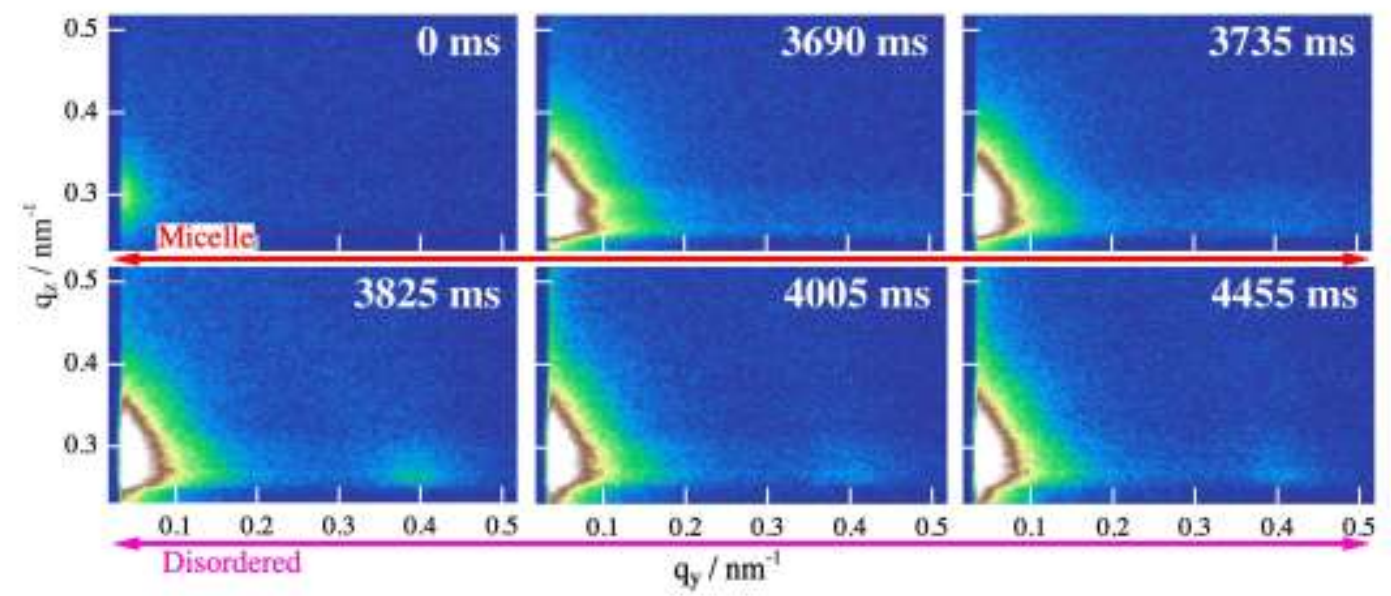

Figure S3. Time-resolved 2D GISAXS images with an incident angle of $0.21^{\circ}$ during spin-coating of PS- $b$-P2VP-1. At $t=3735 \mathrm{~ms}$, a shoulder intensity derived from the micelles was more visible in the in-plane direction at $\mathrm{q}_{\mathrm{z}}=0.288 \mathrm{~nm}^{-1}$. A broad peak corresponding to the ring pattern in thin films was barely visible in the in-plane direction at $t=4005 \mathrm{~ms}$. This peak corresponds to the correlation length of disorderd structures. 


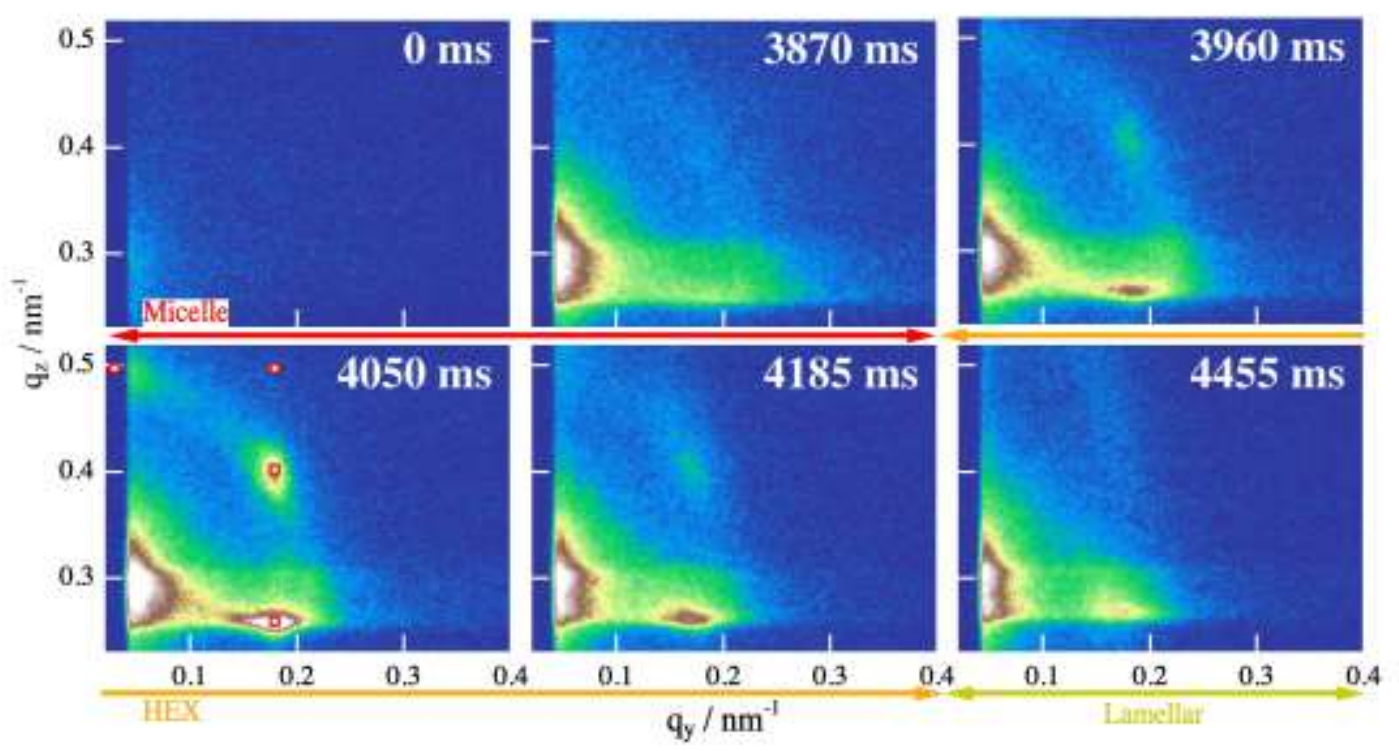

Figure S4. Time-resolved 2D GISAXS images for PS- $b$-P2VP-2. At $t=3960 \mathrm{~ms}$, a scattering spot appeared around $\left(\mathrm{q}_{\mathrm{y}}, \mathrm{q}_{\mathrm{z}}\right)=\left(0.181 \mathrm{~nm}^{-1}, 0.406 \mathrm{~nm}^{-1}\right)$ on a ring pattern. In addition, a weak scattering spot was also observed around $\left(\mathrm{q}_{\mathrm{y}}, \mathrm{q}_{\mathrm{z}}\right)=\left(0.047 \mathrm{~nm}^{-1}, 0.499\right.$ $\mathrm{nm}^{-1}$ ) on the ring pattern, although this was partially blocked by the beam stop. Comparison between the measured 2D image at $4050 \mathrm{~ms}$ and calculated spots estimated from the HEX lattice. (Filled squares denote scattering peaks from the reflected X-ray beams). These spots were not clearly seen at $t=4185 \mathrm{~ms}$. 


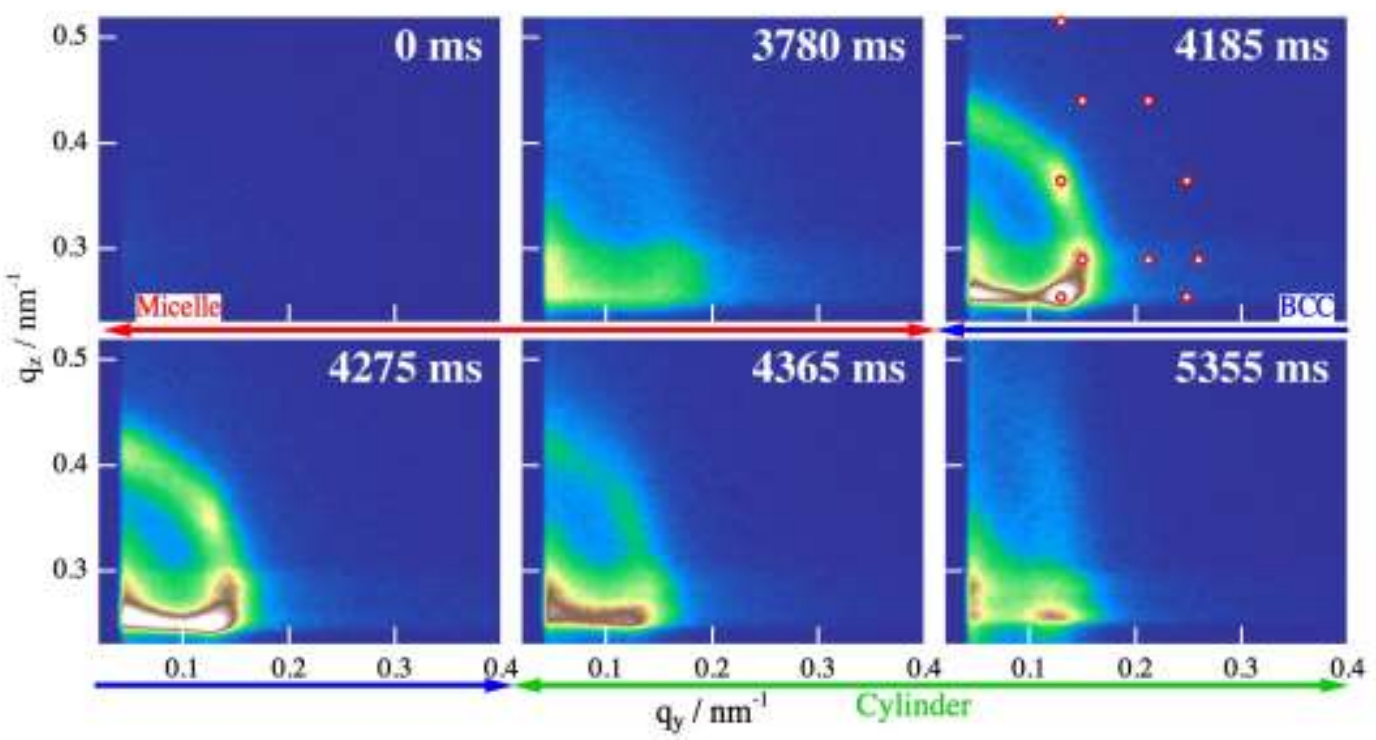

Figure S5. Time-resolved 2D GISAXS patterns for PS- $b-P 2$ VP-3, where the spots correspond to the BCC (110) lattice at $4185 \mathrm{~ms}$. The intensities of the spots weakened at $t=4275 \mathrm{~ms}$. The BCC spots disappeared, and an arc-like pattern corresponding to the correlation lengths of the cylindrical structures was visible at $t=4365 \mathrm{~ms}$.

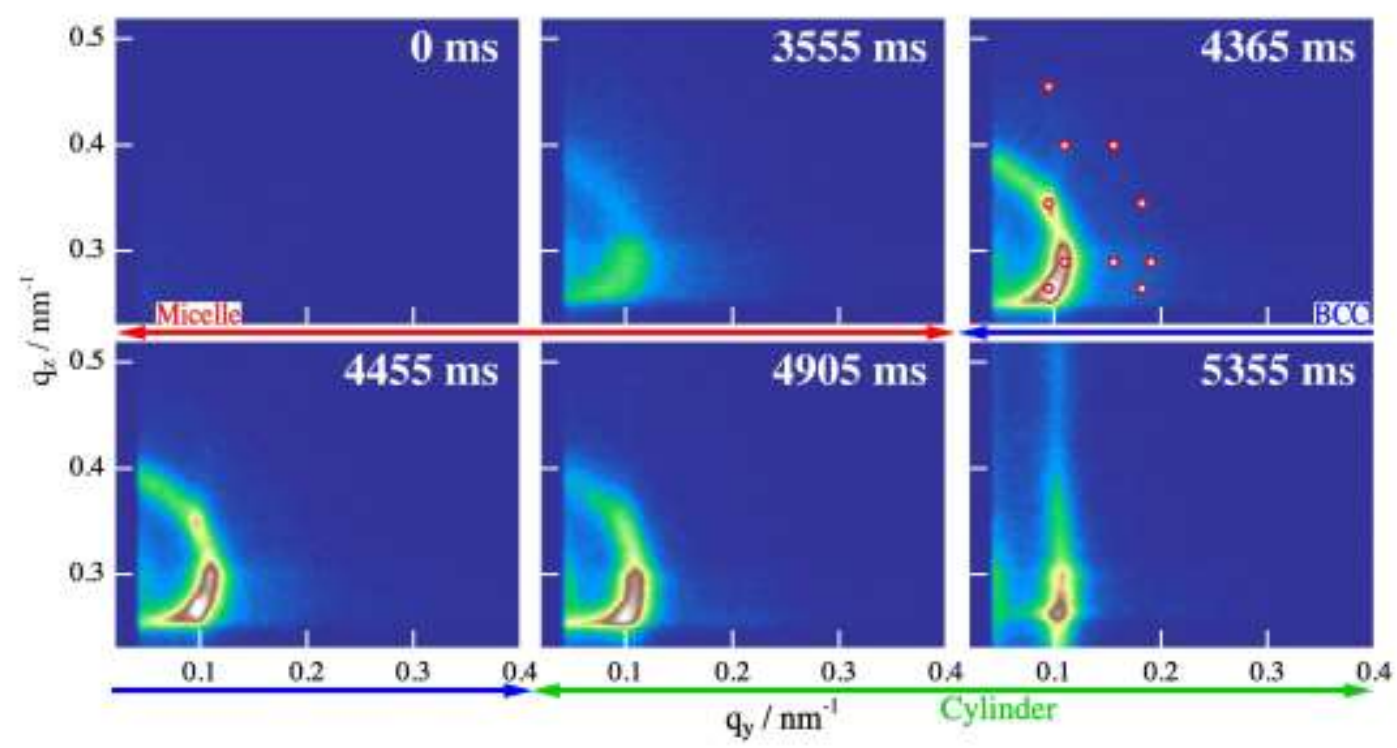

Figure S6. Time-resolved 2D GISAXS images for PS- $b$-P2VP-4, where the spots correspond to the BCC (110) lattice at $4365 \mathrm{~ms}$. The same spots on the ring pattern were 
visible at $t=4455 \mathrm{~ms}$. At $t=4905 \mathrm{~ms}$, the BCC spots disappeared, and an arc-like pattern corresponding to the correlation lengths of the cylindrical structures was visible.

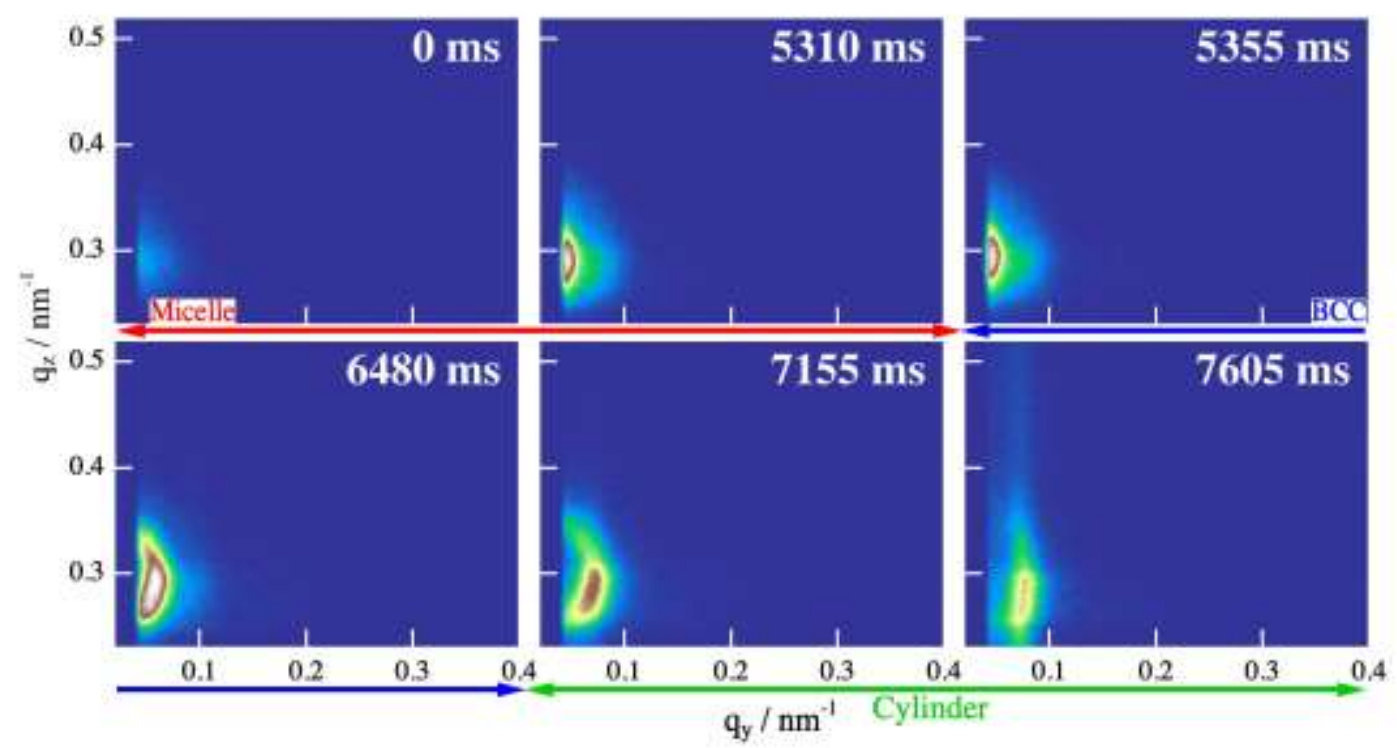

Figure S7. Time-resolved 2D GISAXS images for PS- $b$-P2VP-5. At $t=5355 \mathrm{~ms}$, a ring pattern at a lower $q$ region corresponding to the $d$-spacing of the BCC lattice was visible. An arc-like pattern corresponding to the correlation lengths of the cylindrical structures was visible at $t=7155 \mathrm{~ms}$.

\section{Analysis of scattering spots in hexagonal and BCC lattices.}

The scattering intensity $I(q)$ is obtained by the product of the shape factor $F(q)$ and the lattice factor $Z(q)$. We discuss $Z(q)$ assuming that the shape of the particles of BCC and hcp is the same. Using the Miller indices $(h k l)$, the lattice factors of BCC

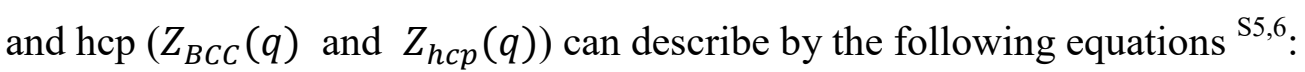

$$
Z_{B C C}(q)=\frac{\sqrt{2} \pi c}{16 D_{d i s}^{3} q^{2}} \sum_{\{h k l\}} m_{h k l} f_{B C C}^{2} L_{h k l}(q)
$$




$$
Z_{h c p}(q)=\frac{\sqrt{2} \pi c}{16 D_{d i s}^{3} q^{2}} \sum_{\{h k l\}} m_{h k l} f_{h c p}^{2} L_{h k l}(q)
$$

where $D_{d i s}$ is the center-to-center distance between neighboring particles, $m_{h k l}$ is peak multiplicities and $L_{h k l}(q)$ is a normalized peak shape function. $c$ is a constant of order unity for crystal lattice. $f_{B C C}$ and $f_{h c p}$ were the symmetry factor that takes into account symmetry-related extinction rules. Each factor can express in the following formula.

$$
\begin{gathered}
f_{B C C}=f\{1+\exp [i \pi(h+k)]+\exp [i \pi(k+l)]+\exp [i \pi(l+h)]\} \\
f_{h c p}=f\left\{1+\exp \left[2 \pi i\left(\frac{h+2 k}{3}+\frac{l}{2}\right)\right]\right\}
\end{gathered}
$$

where $f$ is the atomic scattering factor.

In the BCC lattice, the $f_{B C C}$ value is the same ratio for $h k l$ values. On the other hand, $f_{h c p}$ is different ratio at $h k l$ values in the hcp lattice. For example, $Z_{h c p}$ of the hcp(100) plane is about a $1 / 4$ lower in value than that of the $h c p(110)$ plane. The intensity of the scattering peak arising from the hcp(110) plane in Figure 5 was about 3000 counts in the raw data. The averaged dark value was also about 1800 . The intensity of the hcp(100) plane counted below the dark value was not measurable. Therefore, we were able to observe the hcp(110) surfaces, but not the hcp(100).

\section{Reference}

(1) Babonneau, D. Fit GISAXS: software package for modeling and analysis of GISAXS data using IGOR Pro. J. Appl. Cryst., 2010, 43, 929-936. 
(2) Busch, P.; Rauscher, M.; Moulin, J-F. and Müller-Buschbaum. P. Debye-Scherrer rings from block copolymer films with powder-like order. J. Appl. Cryst., 2011, 44, 370-379.

(3) Kinning, D. J. and Thomas, E. L. Hard-Sphere Interactions between Spherical Domains in Diblock Copolymers. Macromolecules 1984, 17, 1712-1718.

(4) Pedersen, J. S. Determination of Size Distributions from Small-Angle Scattering Data for Systems with Effective Hard-Sphere Interactions. J. Appl. Cryst., 1994, 27, $595-608$.

(5) Förster, S.; Timmann, A; Konrad, M.; Schellbach, C.; Meyer, A.; Funari, S.S.; Mulvaney, P. and Knott, R. Scattering Curves of Ordered Mesoscopic Materials. J. Phys. Chem. B 2005, 109, 1347-1360.

(6) Loose, W. and Ackerson, B. J. Model calculations for the analysis of scattering data from layered structures. J. Chem. Phys. 1994, 101, 7211-7220. 